\title{
Blood Coagulation Indices in Twin Pregnancy Complicated with Preeclampsia
}

\author{
Zhaodong Liu, Huale Zhang, Lichun Chen, Lin Lin and Jianying Yan \\ Department of Gynecology and Obstetrics, Fujian Provincial Maternity and Children Hospital, Affiliated Hospital of Fujian Medical \\ University, Fuzhou, Fujian, P.R. China
}

\begin{abstract}
Objective: To determine the regular changes of blood coagulation indices in twin pregnancy complicated with preeclampsia (PE).

Study Design: Descriptive study.

Place and Duration of Study: Fujian Provincial Maternity and Children Hospital from January 2014 to December 2017. Methodology: A total of 180 twin pregnancies were enrolled in this study, including 40 diagnosed as PE, 50 as severe PE (SPE), and 90 as normal twin pregnancies. The changes of coagulation parameters of twin pregnant women in different gestational states and periods were retrospectively analysed.

Results: During the middle and late pregnancies in all groups, the PT (prothrombin time) decreased, and D-Di (D-dimers) increased gradually compared to early pregnancy $(p<0.05)$. When $D$-Di increased abnormally, adverse pregnancy outcomes increased. APTT (activated partial thromboplastin time) and TT (thromoplastin time) were shortened first and then significantly prolonged $(p<0.05)$. In the normal twin group, FIB was increased gradually from early pregnancy to midlate pregnancy; in the PE group, FIB was increased and then decreased, especially in the sPE group $(p<0.05)$. There was no significant difference in coagulation function in early pregnancy $(p>0.05)$. FIB in SPE group was lower than that in other two groups in late pregnancy. TT in SPE group was higher than that in other two groups. D-Di and APTT in PE group and SPE group were higher than those in normal group $(p<0.05)$. The TT sensitivity was $68.8 \%$ with specificity $72.4 \%$, at cut-off value of $13.48 \mathrm{~s}(\mathrm{p}<0.01)$.

Conclusion: The regular changes of blood coagulation indices in twin pregnancy complicated with PE had great predictive and diagnostic value of preeclampsia.
\end{abstract}

Key Words: Preeclampsia, Twin pregnancy, Coagulation indices.

How to cite this article: Liu Z, Zhang H, Chen L, Lin L, Yan J. Blood coagulation indices in twin pregnancy complicated with preeclampsia. $J$ Coll Physicians Surg Pak 2020; 30(3):276-281.

\section{INTRODUCTION}

Preeclampsia (PE) is a serious complication occurred in the middle and late pregnancy, and seriously affects the prognosis of mother and child. Its pathogenesis is complex and remains unknown.1,2 Patients with PE have abnormal hypercoagulation and hyperfunctional blood coagulation. The imbalance of coagulation and fibrinolysis systems is distinctive feature of PE. Therefore, the detection of these changes of blood coagulation indices for the guidance and prevention of PE has a certain clinical significance. ${ }^{3}$ As the assisted reproductive technology improves, studies have shown that the incidence of twin pregnancies and the incidence of concurrent PE have increased in recent years. Once

Correspondence to: Dr. Jianying Yan, Department of Gynecology and Obstetrics, Fujian Provincial Maternity and Children's

Hospital, Affiliated Hospital of Fujian Medical University,

No.18 Daoshan Road, Gulou District, Fuzhou, Fujian 350001, P.R.China

E-mail: yjianyin172@21cn.com

Received: June 13, 2019; Revised: January 09, 2020;

Accepted: February 03, 2020 the coagulation and fibrinolytic systems are out of balance, the impact on maternal health is even greater because twin pregnancies carry a greater physical burden than single pregnancies. ${ }^{4,5}$ To date, no effective intervention other than termination of pregnancy has been identified, and the challenge of better treatment of PE in twin pregnancies is enormous. Therefore, it is important to identify the appropriate predictors of PE in twin pregnancy for the early prevention and treatment of PE.6,7

The rationale of this study was to explore the dynamic changes in coagulation in twin pregnancies with PE, to identify possible associations with PE development, and to screen for possible predictors to guide the timely and reasonable diagnosis and treatment, improve maternal and infant prognosis.

The objective of the study was to determine the regular changes of blood coagulation indices in twin pregnancy complicated with pre-eclampsia (PE).

\section{METHODOLOGY}

Pregnant women who gave birth to twins were recruited between January 2014 and December 2017 during 
routine obstetric check-ups. The inclusion and diagnostic criteria were based on the ACOG guidelines. ${ }^{8,9}$ Inclusion criteria were twin pregnancies with preeclampsia or severe preeclampsia. Exclusion criteria were twin pregnancies with autoimmune diseases, chronic hypertension, chronic kidney disease, chronic liver disease, hematological diseases, and history of oral contraceptives, and without recent administration of low molecular weight heparin, aspirin and other drugs that affect blood clotting. Randomly selected 90 normal pregnant women with similar gestational age were as the control group. Informed consents were obtained from all participants. This study was a retrospective descriptive study and was reviewed and approved by the Ethics Committee of the Fujian Provincial Maternal and Child Health Hospital.

Participants were divided into three groups: the normal twin pregnancy group, the PE twin pregnancy group, and the sPE twin pregnancy group. These groups were then divided into three subgroups according to the stage of pregnancy [early pregnancy $\leq 12+6$ weeks, midterm 13-27+6 weeks and third trimester 28-41 weeks]. Comparison of each test indicator was performed between and among the groups.

The changes of coagulation function including TT (thromboplastin time), PT (prothrombin time), APTT (activated partial thromboplastin time), FIB and D-Di (D-dimers) were analysed in different pregnant states and during different periods of pregnancy. The coagu-lation function was examined using American Beckman ACL7000 automatic coagulation analyzer and corollary reagents.
All statistical analyses were performed using SPSS 17.0 software. Measurement data were expressed as mean \pm standard deviation $(x \pm s d)$. Homogeneity of variance of each group was performed using Levene' test. If the variance was uniform, one-way ANOVA was used for comparison among groups, and the LSD-t method was used for further comparison between two groups. If the variances were not consistent, the Kruskal-Wallis $\mathrm{H}$ test was used for inter-group comparisons, and Dunnett's T3 method was used for further comparison between the two groups. The area under the curve (AUC) was calculated with the receiver operating characteristic curve (ROC curve), the predictive variables were screened, and the diagnostic parameters (cut-off values) were calculated; $\alpha=0.05$ was used as the test level, and $p<0.05$ was regarded as statistically significant.

\section{RESULTS}

No significant differences in age and pre-pregnancy body mass index were observed among the three groups $(p>0.05)$. No significant differences were seen in the number of gestation days during examination in early pregnancy, middle pregnancy and late pregnancy $(p>0.05)$ Table I.

As the pregnancy progressed, PT gradually shortened, and the differences among the groups were statistically significant $(p<0.05)$. There were no statistical differences between the two groups $(p>0.05)$. In the middle and late pregnancy, PT was shorter in the PE and SPE groups than in the normal pregnancy group, and the differences were statistically significant $(p<0.05)$. From early pregnancy to middle-late pregnancy, no significant differences were

Table I: Comparison of general data of three groups of pregnant women.

\begin{tabular}{l|c|c|c}
\hline & Normal $(\mathrm{n}=90)$ & PE & sPE \\
$(\mathrm{n}=40)$ & & \\
& & $32.26 \pm 4.92$ & $31.62 \pm 5.28$ \\
Age (years) & $31.68 \pm 4.08$ & $20.76 \pm 3.25$ & $21.32 \pm 3.16$ \\
BMl at term $\left(\mathrm{Kg} / \mathrm{m}^{2}\right)$ & $21.24 \pm 3.06$ & $59.85 \pm 15.5$ & $57.82 \pm 15.6$ \\
Early pregnant test day (d) & $56.96 \pm 16.7$ & $172.03 \pm 21.4$ & 0.283 \\
Middle pregnant test day (d) & $166.51 \pm 23.3$ & $241.68 \pm 15.2$ & 0.207 \\
Late pregnant test day (d) & $231.96 \pm 21.9$ & 0.324 & $229.32 \pm 19.3$ \\
\hline
\end{tabular}

Table II: Changes in coagulation function parameters in three groups of pregnant women (x $\pm s d)$.

\begin{tabular}{|c|c|c|c|c|c|c|}
\hline Group & Case (n) & TT (s) & PT (s) & APTT (s) & FIB $(g / L)$ & D-D (mg/L) \\
\hline Normal group & 90 & & & & & \\
\hline Early & 21 & $13.19 \pm 1.01$ & $10.83 \pm 0.60$ & $30.05 \pm 2.85$ & $3.62 \pm 0.66$ & $0.17 \pm 0.13$ \\
\hline Middle & 30 & $12.73 \pm 1.02$ & $10.14 \pm 0.42^{a}$ & $27.06 \pm 1.96 \mathrm{a}$ & $4.42 \pm 0.76$ & $0.56 \pm 0.34 a$ \\
\hline Late & 39 & $12.95 \pm 1.21$ & $9.83 \pm 0.40^{a b}$ & $28.21 \pm 2.52^{\mathrm{ab}}$ & $4.47 \pm 0.96^{a}$ & $0.84 \pm 0.33 \mathrm{ab}$ \\
\hline PE group & 40 & & & & & \\
\hline Early & 14 & $13.05 \pm 1.34$ & $10.85 \pm 0.74$ & $30.25 \pm 3.42$ & $3.66 \pm 0.88$ & $0.20 \pm 0.18$ \\
\hline Middle & 12 & $12.89 \pm 1.10$ & $9.90 \pm 0.44 \mathrm{ac}$ & $27.44 \pm 1.62^{a}$ & $4.48 \pm 0.91 a$ & $0.62 \pm 0.49 a$ \\
\hline Late & 14 & $13.15 \pm 0.97$ & $9.62 \pm 0.33 \mathrm{abc}$ & $29.12 \pm 2.74 \mathrm{bc}$ & $4.35 \pm 0.70 a$ & $1.05 \pm 0.80 \mathrm{abc}$ \\
\hline SPE group & 50 & & & & & \\
\hline Early & 20 & $13.18 \pm 1.22$ & $10.68 \pm 0.62$ & $30.44 \pm 3.05$ & $3.71 \pm 0.70$ & $0.20 \pm 0.19$ \\
\hline Middle & 20 & $13.15 \pm 1.20$ & $9.81 \pm 0.48 \mathrm{ac}$ & $28.02 \pm 1.95 \mathrm{ac}$ & $4.26 \pm 0.82^{a}$ & $0.66 \pm 0.64 a$ \\
\hline Late & 10 & $14.11 \pm 1.32 \mathrm{~cd}$ & $9.44 \pm 0.46 \mathrm{abcd}$ & $29.36 \pm 2.83 b c$ & $3.83 \pm 0.84 \mathrm{~cd}$ & $1.49 \pm 1.32^{\mathrm{abcd}}$ \\
\hline
\end{tabular}

a: $p<0.05$, comparison within the groups, compared with early pregnancy; $b: P<0.05$, comparison within the groups, compared with middle pregnancy; $c: p<0.05$, comparison between the groups, compared with the normal group; $d: p<0.05$, comparison between the groups, compared with PE group. 
Table III (a): AUC for each variable in late pregnancy.

\begin{tabular}{l|c|c|c|c|c}
\hline Test result variables & AUC & Standard error & p-value & \multicolumn{2}{|c}{ Lower limit } \\
\hline TT (s) & & & & 0.750 \\
PT (s) & 0.824 & 0.037 & 0.000 & 0.177 \\
APTT (s) & 0.262 & 0.043 & 0.000 & 0.897 \\
FIB (g/L) & 0.556 & 0.054 & 0.286 & 0.347 \\
DD (mg/L) & 0.278 & 0.046 & 0.000 & 0.187 \\
\hline
\end{tabular}

Table III (b): Predictive value of each predictor of late pregnancy.

\begin{tabular}{|c|c|c|c|c|c|c|c|c|}
\hline \multirow[t]{2}{*}{ Predictor } & \multirow[t]{2}{*}{ Cut-off value } & \multirow[t]{2}{*}{ Sensitivity (\%) } & \multirow[t]{2}{*}{ Specificity (\%) } & \multirow{2}{*}{$\begin{array}{c}\text { Peak of Youden } \\
\text { index }\end{array}$} & \multirow[t]{2}{*}{ OR value } & \multicolumn{2}{|c|}{$95 \% \mathrm{Cl}$} & \multirow[t]{2}{*}{$\mathrm{p}$-value } \\
\hline & & & & & & Lower limit & Upper limit & \\
\hline $\mathrm{TT}(\mathrm{s})$ & 13.45 & 80.0 & 72.5 & 0.525 & 10.545 & 4.511 & 24.651 & 0.000 \\
\hline APTT(s) & 28.25 & 58.0 & 58.8 & 0.168 & 1.249 & 0.613 & 1.892 & 0.590 \\
\hline $\mathrm{DD}(\mathrm{mg} / \mathrm{L})$ & 1.18 & 44.0 & 80.0 & 0.240 & 4.929 & 2.114 & 11.492 & 0.000 \\
\hline
\end{tabular}

observed in TT among the groups ( $p>0.05)$. There were no significant differences between the two groups $(p>0.05)$. In late pregnancy, TT was higher in sPE group than in other two groups, and the differences were statistically significant $(p<0.05)$. From early pregnancy to middle-late pregnancy, APTT was increased and then decreased in all groups, but it was still lower in late pregnancy than in early pregnancy. APTT was higher in PE and SPE groups than in the normal group, and the differences was statistically significant among the groups ( $p<0.05$ ). From early pregnancy to middle-late pregnancy, DD was gradually increased, and the differences were statistically significant $(p<0.05)$. No significant differences were seen in early and middle pregnancy among the groups $(p>0.05)$, while significant differences were observed in late pregnancy $(p<0.05)$. FIB was gradually increased in the normal group during pregnancy, and the differences were statistically significant among the groups $(p<0.05)$. FIB was increased and then decreased in PE and SPE groups, and it was still higher in late pregnancy than in early pregnancy. No

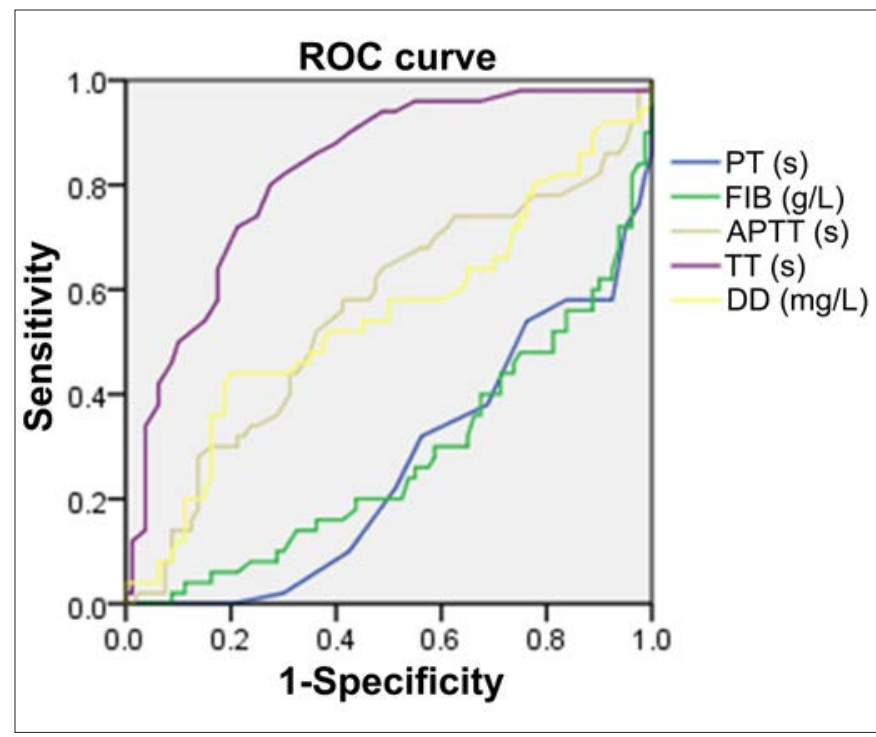

Figure 1: ROC curve of PE predicted by various parameters in late pregnancy. significant differences were observed in comparisons among the groups $(p>0.05)$. FIB was lower in sPE group than in other two groups in late pregnancy, and the differences was statistically significant $(p<0.05)$. Changes in coagulation function indicators are shown in Table II.

There were no obvious predictive values of the predicators in early pregnancy. Taking the ROC curve of PE in middle pregnancy, and the AUC of APTT and TT was 0.674 and 0.576 respectively, which has certain predictive significance, but the diagnostic accuracy was low. The ROC curve is shown in Figure 1. Taking the ROC curve of PE in late pregnancy, TT was an ideal predictor with an AUC of 0.824 and a cutoff value of $13.45 \mathrm{~s}$. TT predicted PE sensitivity of $80.0 \%$, specificity of $72.5 \%$, and odd ratio of 10.545 (Figure 1, Table III).

\section{DISCUSSION}

The heavy uterine cavity pressure in twin pregnancies tends to trigger insufficient placental perfusion, resulting in placental ischemia and hypoxia synthesis and secretion of a large number of inflammatory factors, oxygen-free radicals into the mother's blood circulation, aggravating oxidative stress, and immune damage, triggering vascular endothelium Serious cell damage and dysfunction are more likely to induce hypertensive disorders in pregnancy than single pregnancy, and correspondingly increase the incidence of severe preeclampsia. Besides, twin pregnancies occur earlier, and the condition is more serious, which increases the risk of adverse outcomes such as postpartum hemorrhage and neonatal asphyxia. ${ }^{10,11}$ Therefore, screening and prediction of PE in twin pregnancies has become a widespread concern in clinical practice.

No obvious abnormality was seen in the blood coagulation status of the normal twin pregnancies in early pregnancy. In the middle and late pregnancy, the coagulation system and the fibrinolytic system underwent a series of changes to meet the needs of pregnancy. The content of FIB in the blood was gradually increased, which shortened the PT and APTT. 
Compensatory homeostasis in physiological hypercoagulability and low fibrinolysis returned to prepregnancy status after four weeks of delivery. This hypercoagulation is essential for the integrity of the placental function, delivery and postpartum hemostasis. But it also increases the risk of thrombosis. In this study, PT and APTT of the normal group were significantly shortened in middle and late pregnancy than in early pregnancy, while FIB and D-Di were significantly increased, suggesting the hypercoagulability of the blood.

The dysfunction of the coagulation system and the fibrinolytic system is an important feature of PE. With the progress of pregnancy, PT was gradually shortened in the middle and late pregnancy, and PT was shortened more significantly in PE and SPE groups than in the normal group ( $p<0.05)$. Compared with the normal twin pregnancy group, TT, APTT and D-Di in late pregnancy showed obvious abnormalities in the PE group than in the normal group $(p<0.05)$. PT and APTT are indicators for detecting exogenous and endogenous coagulation systems respectively. D-Di is a specific degradation product of FIB. Its increased plasma content indicated enhanced fibrinolytic activity, and their abnormalities in $P E$ suggested a serious imbalance between the coagulation system and the fibrinolytic system. D-Di was gradually increased in the middle and late pregnancy, and it was significantly higher in the SPE group than in other groups $(p<0.05)$. The abnormal increase of D-Di reflected the secondary fibrinolysis. Therefore, the detection of changes in D-Di level can be used as an auxiliary indicator for the diagnosis of PE, and also determine the severity of the disease and prognosis, providing a basis for twin pregnancy management and clinical diagnosis and treatment.12,13 TT mainly reflects the time of fibrinogen conversion to fibrin, and the content and activity of FIB in the blood. In general, TT and FIB have opposite trends, which is more obvious in the late pregnancy of PE. Pearson correlation analysis showed a significant negative correlation between TT and FIB ( $r=-0.593, p<0.001)$. TT may be an ideal predictor of the onset and severity of PE.

It is well known that PE patients have abnormally increased coagulation substances and clotting activity.14,15 However, the study found that FIB in the PE group was increased and then decreased in the PE group; and was significantly higher in the middle pregnancy than in the early pregnancy, when compared with that in the normal twin pregnancy group. Although FIB was still higher in the late pregnancy than in the early pregnancy, but lower than in the middle pregnancy. This trend was more obvious in the SPE group. The results indicated that with the progression of PE disease, the fibrinolytic system was activated and gradually increased, the consumption of clotting factor FIB was increased, decompensation occurred, and FIB showed a downward trend. If the disease continued to develop, serious complications such as HELLP syndrome appeared. FIB consumption is significantly reduced, leading to DIC and MODS. The reason may lie in that increased liver burden of PE patients in the late pregnancy leads to a decrease in FIB synthesis. On the other hand, the hypercoagulable state and microthrombosis in the circulatory system also consume a large amount of FIB. The coagulation and fibrinolysis systems of PE patients have experienced severe and complex changes, rather than simply increasing the procoagulant substance.16,17

Coagulation function indicators experienced significant changes in the early pregnancy, began to show abnormalities in the middle pregnancy, and had the greatest change in the late pregnancy. These indicators showed greater changes in the severe PE group. The study data showed that TT and APTT were optional predictors in the second trimester, but the diagnostic accuracy was low. In the third trimester of pregnancy, the specificity of DD is higher, but the sensitivity is poor; TT is the most predictive and has a high diagnostic value. In the middle and late pregnancy, TT may be a more reliable indicator for early prediction of PE.

In the current study, the authors focused on PT, D-Di, APTT, TT and FIB because they were classical indicators in blood coagulation system and they often presented with significant differences between twin pregnancy with PE and singleton. However, the recent reports mostly limited to singleton. Due to the heterogeneous pathophysiology and genetic factors, no single indicator is sufficient for the prediction and diagnosis of twin pregnancies with PE. 18 Few studies identified that serum markers ubiquitously used in singleton pregnancy may be useful in twin pregnancy with PE. ${ }^{19}$ But their clinical application and analysis have never been fully demonstrated to date.

At present, most of the researches on the predictive indicators of PE at home and abroad are retrospective studies on singleton pregnancy. The coagulation indicators with predictive value in these studies on PE included TT, APTT, and D-Di.20-23 Han L et al. performed a retrospective study in 174 women with singleton pregnancy (53 cases of mild PE, 42 cases of severe PE, and 79 cases of normal pregnant women). 24 The ROC curve analysis showed that TT had the highest predictive value for PE (AUC: 0.743 ) with a cut-off value of $12.65 \mathrm{~s}$, which is recommended as a potential indicator for early monitoring of the onset and severity of PE. In this study, the cut-off value of TT in late pregnancy was $13.45 \mathrm{~s}$, which was higher than that of Han $L$ et al. The reason may lie in the difference in preeclampsia between singleton and twin pregnancies. The changes in blood coagulation indicators were more obvious and the condition was more serious in twin pregnancies with PE of late pregnancy.

The present study has some shortcomings. The sample size of twin pregnancies with and without PE was very 
small due to the low prevalence of PE. In the foreseeable future, a much larger sample size is needed to unveil the underlying mechanism of changes of of blood coagulation indices in twin pregnancy with PE.

\section{CONCLUSION}

Normal twin pregnancies have a physiological hypercoagulable state in the middle and late pregnancies. When combined with PE, systemic arteriolar spasm and vascular endothelial cells are severely damaged, which may cause abnormalities of the internal and external coagulation system, abnormal blood hypercoagulability, consumption of blood coagulation factors such as platelets and fibrinogen. The reason may be the difference between preeclampsia in singleton and twin pregnancies. The change of coagulation index of PE in late pregnancy with twin pregnancy is more obvious and the condition is more serious.

\section{ETHICAL APPROVAL:}

This study has been reviewed and approved by the Ethics Committee of the Fujian Provincial Maternal and Child Health Hospital.

\section{PATIENTS' CONSENT:}

Informed consents were obtained from all participants.

\section{CONFLICT OF INTEREST:}

Authors declared no conflict of interest.

\section{AUTHORS' CONTRIBUTION:}

JY: Lead and conceived the project.

ZL: Contributed to design articles, collected and analysed data, and authored the manuscript.

HZ, LL: Contributed to collect and analyse data.

LC: Helped perform the analysis with constructive discussions.

\section{FUNDING:}

Fujian Provincial Maternity and Children Hospital Science and Technology Innovation Start-up Fund (YCXM 18-28) Fujian Science and Technology Leading Project (2018Y0005).

\section{REFERENCES}

1. Duhig K, Vandermolen B, Shennan A. Recent advances in the diagnosis and management of pre-eclampsia. F1000 Res 2018; 7:242

2. Peres GM, Mariana M, Cairrao E. Pre-eclampsia and eclampsia: An update on the pharmacological treatment applied in Portugal. J Cardiovasc Dev Dis 2018; 5:E3.

3. Pinheiro MB, Gomes KB, Dusse LM. Fibrinolytic system in preeclampsia. Clin Chim Acta 2013; 416:67-71.

4. Li Y, Zhao S, Yu Y, Ma C, Zheng Y, Niu Y, et al. Risk factors associated with pre-eclampsia in pregnancies conceived by ART. Reprod Biomed Online 2019; 39:969-975.
5. Li GT, Xu HM, Li GR. Prophylactic uterine compression sutures for addressing potential postpartum hemorrhage. J Obstet Gynaecol Res 2020; 46:547-9.

6. Pennington KA, Schlitt JM, Jackson DL, Schulz LC, Schust DJ. Preeclampsia: Multiple approaches for a multifactorial disease. Dis Model Mech 2012; 5:9-18.

7. Vilchez G, Patel N, Maulik D. Postpartum hemorrhage refractory to uterotonics. BJOG 2019 [Epub ahead of print].

8. Westberg SM, Pereira C, Rosdahl R, Do A, Moon JY, Melnik T. Management of hypertension in pregnancy: A descriptive report of two clinic practices. Hypertens Pregnancy 2020; 39:43-7.

9. Ulrich CL, Utarnachitt RB, Danielson K, Pieri A, Whitley S, Latimer AJ. Postpartum hemorrhage with cardiorespiratory collapse transported from a rural hospital. Air Med J 2019; 38: 440-1.

10. Weiner E, Feldstein O, Schreiber L, Grinstein E, Barber E, Dekalo $A$, et al. Placental component and pregnancy outcome in singleton versus twin pregnancies complicated by preeclampsia. Fetal Diagn Ther 2018; 44:142-8.

11. Spencer SPE, Lowe SA. Ergometrine for postpartum hemorrhage and associated myocardial ischemia: Two case reports and a review of the literature. Clin Case Rep 2019; 7:2433-42.

12. Xu JY, Zhou ML, He YH, Chen DQ. Characteristics and clinical significance of serum D-dimer level before and after delivery in women with advanced maternal age. Zhonghua Yi Xue Za Zhi 2019; 99:2768-72.

13. Thangaraju P, Varthya SB, Venkatesan S. Low-dose oxytocin a safe strategy in postpartum hemorrhage. Indian J Pharmacol 2019; 51:355-6.

14. Ortner CM, Krishnamoorthy V, Neethling E, Flint M, Swanevelder JL, Lombard C, et al. Point-of-care ultrasound abnormalities in late-onset severe preeclampsia: prevalence and association with serum albumin and brain natriuretic peptide. Anesth Analg 2019; 128:1208-16.

15. Nsangamay T, Mash R. How to improve the quality of care for women with postpartum haemorrhage at Onandjokwe Hospital, Namibia: Quality improvement study. BMC Pregnancy Childbirth 2019; 19:489.

16. Gungor ZB, Ekmekci H, Tuten A, Toprak S, Ayaz G, Caliskan O, et al. Is there any relationship between adipocytokines and angiogenesis factors to address endothelial dysfunction and platelet aggregation in untreated patients with preeclampsia? Arch Gynecol Obstet 2017; 296:495-502.

17. Majluf-Cruz K, Anguiano-Robledo L, Calzada-Mendoza CC, Hernandez-Juarez J, Moreno-Hernandez M, DominguezReyes VM, et al. Von Willebrand disease and other hereditary haemostatic factor deficiencies in women with a history of postpartum haemorrhage. Haemophilia 2020; 26:97-105.

18. Kim YR, Jung I, Heo SJ, Chang SW, Cho HY. A preeclampsia risk prediction model based on maternal characteristics and serum markers in twin pregnancy. $J$ Matern Fetal Neonatal Med 2019; 1-6.

19. Sibai BM, Koch MA, Freire S, Pinto e Silva JL, Rudge MV, Martins-Costa S, et al. Serum inhibin A and angiogenic factor 
levels in pregnancies with previous preeclampsia and/or chronic hypertension: are they useful markers for prediction of subsequent preeclampsia? Am J Obstet Gynecol 2008; 199:e1-9.

20. Gyselaers W. Maternal venous hemodynamic dysfunction in proteinuric gestational hypertension: Evidence and Implications. J Clin Med 2019; 8:E335.

21. Gong JM, Shen Y, He YX. Reference intervals of routine coagulation assays during the pregnancy and puerperium period. J Clin Lab Anal 2016; 30:912-7.

22. Wang M, Lu S, Li S, Shen F. Reference intervals of D-dimer during the pregnancy and puerperium period on the STA-R evolution coagulation analyzer. Clin Chim Acta 2013; 425 : 176-80.

23. Gonzalez-Fiol A, Eisenberger A. Anesthesia implications of coagulation and anticoagulation during pregnancy. Seminars Perinatolo 2014; 38:370-7.

24. Han L, Liu X, Li H, Zou J, Yang Z, Han J, et al. Blood coagulation parameters and platelet indices: Changes in normal and preeclamptic pregnancies and predictive values for preeclampsia. PLoS One 2014; 9:e114488.

......... 